\title{
La justicia relacional como círculo virtuoso
}

\author{
Relational Justice as Virtuous Circle
}

ANTONIO MÁRQUEZ PRIETO

UNIVERSIDAD DE MÁLAga

Artículo recibido: 30 junio 2013

Solicitud de revisión: 08 octubre 2013

Artículo aceptado: 22 octubre 2013

\section{Resumen}

La idea de justicia relacional, que conecta el mundo del Derecho con el giro relacional de la Sociología, de Pierpaolo Donati, al tiempo que incorpora la noción económica de reciprocidad de Luigino Bruni, pretende analizar la realidad jurídica en base a tres elementos, propios de un enfoque relacional interdisciplinar: institucionalidad, reciprocidad y socialidad. Por ello, el libro Le nuove virtú del mercato nell'era dei beni comuni (Bruni, 2012) constituye una oportunidad óptima para presentar la justicia relacional, precisando su sentido, en conexión con la idea de virtud y, más concretamente, con la buena fe. Este estudio alude, pues, a la justicia relacional, como categoría concreta de justicia, como nota presente -o ausente- en la relación jurídica, y como explicación del cierre del círculo virtuoso de la justicia (o, por el contrario, el círculo vicioso de la injusticia) en la relación.

Palabras clave: justicia relacional, reciprocidad, buena fe, virtud, socialidad

\begin{abstract}
The idea of relational justice, which connects the world of Law with the relational turn of Sociology, of Pierpaolo Donati, while incorporating the economic notion of reciprocity of Luigino Bruni, attempts to analyze the legal reality based on three elements, typical of an interdisciplinary relational approach: institutionality, reciprocity and sociality. Therefore, the book Le nuove virtu del mercato nell'era dei beni comuni (Bruni, 2012) is a unique opportunity to present relational justice, specifying its meaning, in connection with the idea of virtue and, more specifically, with good faith. This study refers, then, to relational justice, as a particular category of justice, as a present - or absent - feature in the legal relationship, and as an explanation for the closing of the virtuous circle of justice (or, conversely, the vicious circle of injustice) in the relationship.
\end{abstract}

Keywords: relational justice, reciprocity, good faith, virtue, sociality. 


\section{JUSTICIA RELACIONAL, COMO DINÁMICA ENTRE INSTITUCIONALIDAD Y RECIPROCIDAD}

Aunque «la relacionalidad, en sentido moderno, ha entrado en las ciencias sociales sobre todo con Durkheim», ${ }^{1}$ el primero en analizar la relación social en base a sus componentes fue Talcott Parsons, cuyas afirmaciones sobre el fenómeno social se expresaban en términos de relacionalidad de sus elementos, interdependencia entre los componentes de la relación social (García Ruiz, 2000). Según dicho autor, la idea de interdependencia manifestaba no sólo dicha interrelación, sino también el efecto emergente de la misma (aunque sólo a un cierto nivel de complejidad de relaciones). Para expresar la relacionalidad entre los componentes de un sistema social propuso el «esquema AGIL», acrónimo formado con las iniciales en inglés de cuatro funciones o dimensiones: A (adaptation), referido a los medios o recursos; $G$ (goal-attaintment), que expresa los fines y los objetivos concretos en cada situación; I (integration), es decir, normas reguladoras de las acciones o relaciones; L (latency), que indica el modelo de valor latente que subyace al sistema. Esos cuatro componentes pueden, de manera más clarificadora, contemplarse como dos ejes: el eje referencial (L-G), como relación entre valores básicos y determinados objetivos situacionales - es decir, la relación entendida como refero-; y el eje estructural (A-I), que representa los medios conectados con las normas de comportamiento - es decir, la relación entendida como religo-. En esta duplicidad de ejes o dimensiones de la relación social -así como en el mencionado efecto emergente de la misma- existe un potencial relacional, no desarrollado por Parsons, ${ }^{2}$ pero que ha posibilitado en gran medida a otros, posteriormente - es significativo el caso de Donati-, llevar a cabo el llamado «giro relacional» de la Sociología: (Donati, 2006: 113). ${ }^{3}$

1 Añadiéndose que su funcionalismo específico define los entes sociales por su función, en vez de por su finalidad (es decir, en base a consideraciones objetivas y no subjetivas ni intencionales); explicitándose, a partir de aquí, dos reglas específicas: «poner en relación las partes de las sociedad con el todo, y relacionar cada una con las demás, según sus funciones» (Donati, 2006: 138-139).

2 «Los sistemas de acción tienen propiedades que emergen sólo a un cierto nivel de complejidad en las relaciones entre las unidades singulares» (Parsons, 1937: 739).

3 Acerca de la perspectiva sistémica funcional, el autor reconoce (Donati, 2006: 111-112) que la sociología relacional no nace en el vacío - en referencia a la misma-, habiendo sido dicho enfoque el que más ha enfatizado el papel de las relaciones y el que más ha usado la noción de red. «Pero, desafortunadamente, ofrece una visión demasiado reducida (únicamente funcional) de la relación social. Además, trata el concepto de red en clave estructuralista, lo que supone otra reducción inaceptable». En otro lugar de la misma obra, aclara: «Pero es sobre todo en Alemania, con Georg Simmel, Max Weber, Leopold von Wiese, Martin Buber y la fenomenología de Edmund Husserl, donde se completa el giro relacional en las ciencias sociales. Giro relacional significa la transición de un pensamiento entitativo - que razona y observa según entidades, tratando de conocer aquello que 
La sociología relacional presupone el «giro relacional» de la sociedad que, aunque ha aparecido con la modernidad, va ciertamente más allá de ésta.Trata, por eso, de desarrollar la visión relacional de la sociedad que, iniciada por Marx, Weber y Simmel, ha encontrado en estos autores sólo una primera y parcial interpretación

En realidad, Donati, aunque declara que el giro relacional «se ha llevado a cabo en gran parte bajo la enseñanza del funcionalismo» (Donati, 2006: 138), se separa del mismo en aspectos cruciales, uno de los cuales consiste en la explicación del cambio social.Así, tomando como referencia el esquema AGIL de Parsons, Donati atribuye la mayor importancia al componente de latencia (L), a diferencia de aquél, que consideraba protagonista a la capacidad de adaptación del sistema (A), desde el punto de vista de la eficacia. Para Donati, «lo que realmente introduce novedad en la vida social es la creatividad de los seres humanos que asignan un nuevo significado a lo ya existente o a lo porvenir» (García Ruiz, 2006: 41). Y así explica la relación social en base a tres semánticas:

a) la semántica referencial (o también simbólica, intencional, significativa), que se corresponde con el eje o dimensión referencial de la relación (L-G): desde este enfoque la relación se entiende como un refero, un referir una realidad a otra, una atribución de sentido, en términos de significado e intenciones ${ }^{4}$

b) la semántica estructural (de generación de vínculos y dependencias mutuas), que se corresponde con el eje o dimensión estructural (A-I): desde este punto de vista la relación es religo, es decir, «sujeción, conexión, atadura, condicionamiento recíproco, estructura, que es al mismo tiempo vínculo y recurso, de carácter personal e impersonal» (Donati, 2006: 93)

c) la semántica generativa: esta tercera semántica explica cómo, de los sujetos y agentes que entran en relación, emerge, se genera, algo distinto que, en realidad, es lo que propiamente se puede llamar relación. ${ }^{5}$

existe en sí y por sí- a un pensamiento relacional, que opera distinguiendo reflexivamente por relaciones, y se orienta de algún modo a la construcción del propio objeto: aquello que existe-enrelación» (pp. 65-66).

4 En referencia al funcionalismo representado por Luhmann, Donati critica la visión de un funcionamiento social mecánico, automático, según los mecanismos de la evolución sistémica, confiando todo a sistemas técnicos que eviten a las personas el esfuerzo de activar recursos latentes y personalizados para solucionar problemas y patologías sociales, «mientras que para la sociología relacional [esos mecanismos] están mediados por la conciencia o conversación interior de las personas humanas» (Donati, 2006: 160).

5 Ésta es la definición que Donati ofrece de relación social (2006: 95): «Desde el punto de vista de las ciencias sociales, podemos decir que la relación social es aquella referencia -simbólica o intencional- 
En este sentido, puede afirmarse que «la emergencia de lo social», aun siendo «consecuencia de la interdependencia de sus componentes [...] contiene siempre una novedad no previsible porque su contenido específico no está presente en sus componentes considerados por separado sino que sólo comparece cuando se establece, se actualiza o se recupera la relación entre ellos» (Donati, 2006: 23).

Estas consideraciones, acerca del enfoque relacional sociológico y esta triple semántica de Donati, son las que han dado lugar (con ciertas adaptaciones) a la propuesta de la justicia relacional, interesando aquí resaltar sintéticamente que la misma analiza el problema de la justicia en la relación interpersonal, considerando, en este sentido, que la idea de justicia encuentra explicación desde la noción de relación, que es vínculo recíproco, es decir, vinculación (o institucionalidad) y reciprocidad. ${ }^{6}$ La justicia es su efecto emergente si nos situamos en una relación o sistema jurídicos, cuyas referencias, internas y externas, se orientan hacia la misma de forma intencional. Puesto que, desde la concepción de relación, la justicia emergente está ligada a la justicia intencional. ${ }^{7}$

Concretamente la morfogénesis de la justicia tiene lugar a través de una dinámica bilateral en torno a dos ejes o ámbitos: ${ }^{8}$ el de institucionalidad, o vinculación (generación de vínculos y dependencias mutuas), que en estado inicial o germinal es, principalmente, «aceptar y corresponder»; y el de reciprocidad (de relación entre valores y motivaciones u objetivos), que en estado inicial es «dar lo propio», "promover lo otro» (tomando la iniciativa de una sucesiva cadena que ha de funcionar de forma libre y plural). ${ }^{9}$ Nótese que los dos mencionados ámbitos o aspectos de la relación jurídica (institucionalidad y reciprocidad) responden paralelamente a los dos ejes de Donati

que conecta sujetos sociales en la medida en que actualiza o genera un vínculo entre ellos, es decir, en cuanto expresa su acción recíproca. Ésta consiste en la influencia que los términos de la relación tienen el uno sobre el otro y en el efecto de reciprocidad emergente entre ellos».

6 Ver nuestra propuesta (Márquez-Prieto, 2008: 42 ss.) sobre la aplicación del enfoque relacional al Derecho, como intento de superación de la debilidad de la idea de justicia, tras el desbordamiento de la modernidad.

7 Aludiendo, paralelamente, a los niveles intencional y emergente de la relación (Donati, 2006: 54).

8 Vid. también, de forma aclaratoria sobre esta dinámica entre institucionalidad y reciprocidad, CaroGándara, R. (2013: 11-67).

9 Resulta imprescindible anotar que esta relación de bilateralidad ha de ser simétrica para poder funcionar adecuadamente. Sin embargo, en muchas de las situaciones sociales las partes intervinientes se relacionan de forma asimétrica y desigualitaria. Por lo que un elemento de primera relevancia de esta visión de la justicia social es su carácter necesariamente emancipador, o liberador, de la persona, respecto de las estructuras sociales deshumanizantes, de subordinación o de dominación. En este sentido, la relacionalidad exige la inserción, la inclusión en una relación propiamente interpersonal, un ajuste (autónomo o heterónomo) de lo asimétrico con lo horizontal, de la dependencia con la interdependencia por medio del «expediente de inclusión» (Márquez-Prieto, 2008: 52-53). 
(estructural y referencial, respectivamente), que a su vez se apoya en el esquema AGIL de Parsons.

Ignorar el ámbito de la reciprocidad, es decir, el del comportamiento dinámico, interactivo, libre y plural de los sujetos, significa caer en el error de enfocar lo jurídico en base únicamente a las normas, convertidas supuestamente en auto-referenciales. Es preciso recordar que tantas veces el Derecho, desligándose del binomio en el que aparece conectado a la justicia, es pensado restrictivamente como norma y, al mismo tiempo - pero de forma incoherente - llega a caer en la tentación de suplantar a la justicia. Es como si el Derecho, en cuanto ordenamiento jurídico, huyera del posible fracaso de tener que atender a la justicia en las relaciones jurídicas. Precisamente porque la justicia, ya para Aristóteles, asumía su significado, siempre dentro de una relación, respecto de los demás (2001: 1103b). Ciertamente el Derecho no puede prescindir del elemento de obligatoriedad, de la garantía de la fuerza. Pero de alguna forma debe estar dispuesto también a perder la fuerza, a no ser (su fuerza). El Derecho debe encontrarse con la oportunidad -o ausencia- del don - en función de la pluralidad-, hacerse compatible con la dinámica libre que es capaz de acoger a la justicia relacional. Es decir, buscar la justicia, no sólo desde la institucionalidad, sino también desde la reciprocidad. Ir más allá de lo establecido, de lo ordenado, de lo pactado, de lo mandado, de lo obligatorio, de lo exigible (ir al Derecho inorganizado, inordenado). ${ }^{10}$ Si reducimos el Derecho a lo normativo y a su cumplimiento, queda fuera de él una valiosa dinámica jurídica autónoma, en el sentido de «movida, no por el cumplimiento de la norma», sino «automotivada». Una libertad plural de actuar colectivamente, conforme a lo justo, considerado, como tal, necesario, al menos como conciencia (no necesariamente exigible), pero como conciencia relacional, plural. Un etbos que no es ética, en la medida en que el agente jurídico es consciente de su juridicidad.

En definitiva, si la institucionalidad no está abierta a la reciprocidad, se queda en unilateralidad. En puridad, ello no significa que para el Derecho la relación no exista. Pero la considera unilateralmente, viendo en ella sólo su contenido, de manera unidireccional: el derecho de uno respecto del otro; el deber de uno para con el otro. No ve la relación interactiva. Para ello es preciso introducir verdaderamente el enfoque de reciprocidad: el juego continuo y dinámico entre los sujetos de la relación, es decir, la relación propiamente. Eso hace que también lo institucional y lo recíproco se autoadapten, puesto que la reciprocidad no es sólo un aspecto o elemento de la relación,

10 Gurvitch (1932: 49) propone la primacía del Derecho social inorganizado sobre el Derecho social organizado. 
sino también el aspecto que sugiere que exista reciprocidad entre los dos elementos (institucionalidad y reciprocidad). Y, de forma paralela, la institucionalidad hace que la reciprocidad no sea sólo motivación, sino también responsabilidad. En síntesis, pues, aunque el Derecho tenga en cuenta la alteridad, necesita también estar abierto a la alteridad recíproca, que, sin embargo, y por otro lado, merced a su carácter libre, plural y heterogéneo, plantea el problema de la «tragedia de los comunes».

\section{SOCIALIDAD Y BIENES COMUNES}

En referencia al problema aludido, Luigino Bruni (2012: 31), tras afirmar que «el centro de la ciencia de la economía y del mercado lo ocupan hoy los bienes comunes, que son y serán los bienes de los que dependerá la calidad de la vida y probablemente la supervivencia del planeta», añade que «en el mundo de los bienes comunes también personas individualmente virtuosas pueden determinar, de forma no intencional, tragedias colectivas, si no razonamos desde ya en términos de "nosotros", de modo relacional» (31-32). Este planteamiento nos sitúa ante el problema de la cooperación social, respecto al cual las distintas estrategias individuales de actuación, así como la interacción entre las mismas, vienen siendo ilustradas, de forma muy sugerente, con la ayuda de la teoría de juegos. Ciertamente la teoría de juegos parte de una simplificación de la realidad, de un modelo idealizado, que presenta limitaciones si se pretende llegar a conclusiones normativas, o atribuir un carácter definitorio más o menos cerrado a aproximaciones descriptivas; pudiendo ser, por otro lado, muy reveladora para la comprensión de situaciones sociales desde un punto de vista paradigmático (Stanford Encyclopedia, 2004). Es así, a título ilustrativo, como puede resultar útil en este lugar, para la mejor comprensión de la propuesta de justicia relacional. En este sentido conviene analizar qué situación, o qué interpretación de la realidad, reconstruye cada juego, siendo también posible la comparación entre unos y otros.

Si analizamos el problema que plantea la cooperación social en las relaciones sociales (relaciones de trabajo, de mercado...), es necesario aludir a los dos juegos más conocidos al respecto: el dilema del prisionero y la caza del ciervo. En cuanto al primero, el más famoso de los juegos de «suma no cero", su matriz de pagos, o de recompensas asignadas a los jugadores, puede cambiar según el autor que se consulte (Martínez-Coll, 1986; Baird y otros, 1994).Y, de hecho, la puntuación que marca las recompensas se corresponde con el número de años de cárcel a que pueden ser condenados dos sospechosos (prisioneros) de haber cometido juntos un delito. La posible condena 
variará dependiendo de que decidan cooperar entre sí (silenciando los dos la comisión del delito), con lo que conseguirían una pena bastante inferior, o no cooperar (confesando) viendo aumentada su condena dependiendo de que confiesen ambos o sólo uno.

\begin{tabular}{llc}
$\begin{array}{l}\text { Conducta de los jugadores: } \\
\text { Ni A ni B confiesan } \\
\text { (cooperan entre sí) }\end{array}$ & $\begin{array}{l}\text { Cada uno es condenado a } 1 \\
\text { año de cárcel }\end{array}$ & $\begin{array}{l}\text { Puntuación o } \\
\text { recompensa: }\end{array}$ \\
$\begin{array}{l}\text { A silencia, B confiesa (B } \\
\text { defrauda a A) }\end{array}$ & $\begin{array}{l}\text { A es condenado a } 10 \text { años y } \\
\text { B queda libre }\end{array}$ & A=1, B=4 \\
$\begin{array}{l}\text { Ambos confiesan (ambos se } \\
\text { defraudan) }\end{array}$ & $\begin{array}{l}\text { Cada uno es condenado a } 6 \\
\text { años }\end{array}$ & A=2, B=2 \\
$\begin{array}{l}\text { A confiesa, B silencia } \\
\text { (A defrauda a B) }\end{array}$ & $\begin{array}{l}\text { A queda libre y B es } \\
\text { condenado a } 10 \text { años }\end{array}$ & A=4, B=1 \\
\hline
\end{tabular}

Así pues, la matriz de pagos quedaría así:

\begin{tabular}{lcc} 
A/B & Coopera & No coopera \\
\hline Coopera & 3,3 & 1,4 \\
No coopera & 4,1 & 2,2 \\
\hline
\end{tabular}

En este juego existe sólo una situación de equilibrio (equilibrio de Nash), en el lugar donde ambos jugadores manifiestan una actitud de no cooperar con el otro, a pesar de que, según puede verse, la recompensa conjunta sería superior si decidieran cooperar entre sí. Lo que sucede es que cada uno de los prisioneros trata de maximizar su beneficio, sin preocuparse por el resultado que el otro prisionero obtenga. Cada jugador, de hecho, ante la desconfianza de lo que el otro pueda hacer, está más incentivado a defraudar al otro, incluso tras haberse prometido entre sí la cooperación (éste es el dilema).

El segundo de los juegos aludidos, la caza del ciervo, debe su nombre a un relato de Rousseau, que describe la situación en la que se encuentran dos 
cazadores, que deben elegir entre cazar juntos un ciervo o cazar por separado una liebre (Rousseau, 1754). El ciervo constituye una pieza de mucho mayor interés que la liebre, pero exige coordinación entre ambos cazadores, pudiendo representarse así su matriz de pagos:

\begin{tabular}{lcc} 
A/B & Ciervo & Liebre \\
Ciervo & 4,4 & 0,3 \\
Liebre & 3,0 & 2,2 \\
\hline
\end{tabular}

Para algunos autores (Cortázar, 1997; Janssen, 2001; Skyrms, 2007) el juego de la caza del ciervo explica el fenómeno relacionado con la cooperación social -o la ausencia de ella- de forma más completa que el dilema del prisionero. Efectivamente es más completo el juego de la caza del ciervo en la medida en que presenta no una, sino dos situaciones de equilibrio posible: la opción por la caza de la liebre por parte de ambos y la decisión de ambos cazadores de mantenerse en la coordinación conjunta para la caza del ciervo, siendo esta segunda posibilidad la que mayor recompensa comporta. Pero en ambos puede apreciarse con crudeza la llamada «tragedia de los bienes comunes», según el título de un artículo de Hardin (1968), es decir, «una tensión dramática entre la libertad de los individuos y la destrucción de los recursos comunes» (Bruni, 2012: 74). Y precisamente para superar esta tragedia Luigino Bruni propone entrar en la «racionalidad del nosotros» (2012: 92), puesto que tanto un juego como otro muestran que la cooperación social se consigue cuando los jugadores pasan de la lógica individual a la lógica de la relación, es decir, cuando piensan cuál será el mejor resultado para el conjunto. Es eso lo que permite de verdad entrar en la relación -en su mentalidad y en su dinámica-, para lo cual adquiere particular importancia la idea de virtud. Con independencia de que en el siguiente apartado se retome el argumento de la virtud, interesa aquí apuntar el importante papel que, al respecto, Bruni (2012: 93-98) atribuye a la cultura, y por tanto a la sociedad civil (socialidad en su conjunto), como lugar donde nacen las motivaciones, los valores y las preferencias de las personas.

Conviene aquí igualmente, al hilo de lo anterior, y en relación a la justicia relacional, recordar que la misma se explica en base al juego dinámico entre tres elementos (institucionalidad, reciprocidad y socialidad), para concretar ahora el papel del tercer elemento, en la medida en que no ha sido aún directamente abordado: la socialidad, es decir, la relación en sí misma, en la 
medida en que se va continuamente haciendo, así como el conjunto o red de relaciones interconectadas. Así pues, si la relación, o socialidad, adquiere importancia -como se dice en el siguiente apartado-, como contexto de las motivaciones y de la virtud - también como criterio de la buena fe y de la justicia, vid. infra-, procede destacar aquí, concretamente, su relevancia como contexto social en el cual tiene lugar la dinámica bilateral de interacción entre los ámbitos de institucionalidad y de reciprocidad; ese juego jurídico entre norma y comportamiento, que necesariamente se desarrolla en un marco de relaciones jurídicas y sociales en general.

Es en este sentido evidente que el Derecho pertenece al ámbito de la socialidad. Ello comporta la constatación de que la relación jurídica es ante todo relación, y que no importa demasiado aclarar si la socialidad puede con propiedad integrarse en el Derecho, ya que es, al contrario, el Derecho el que se integra en la socialidad. La misma socialidad a la que pertenecen los distintos ámbitos abordados por las distintas ciencias sociales. Es, pues, útil, este tercer elemento para abrir el Derecho a la interdisciplinariedad -a la globalidad, a la complejidad-, que viene facilitada por encontrarse en un mismo espacio (socialidad) las diversas disciplinas. Referida, pues, directamente a la relación, no sólo entre sus sujetos o protagonistas, sino también entre sus aspectos, es la socialidad lo que impulsa a la reciprocidad a salir de sí misma y hacerse institucionalidad (porque de alguna forma está ya presente en la reciprocidad y en la institucionalidad). Este tercer elemento es también pregunta, cuestionamiento, crítica, criterio, conciencia, sobre la justicia y la injusticia en la relación.

Lo podemos llamar justamente socialidad porque la relación tiene lugar en el espacio social, donde adquiere su sentido. Así, por ejemplo, la relación entre Administración y administrado (que en sí misma aparece a veces sólo como jerarquía) encuentra su sentido (su función y su equilibrio) en un contexto de sociedad. Este tercer elemento, en cuanto ámbito social, es, de forma más completa, la relación in fieri (en la medida en que se está continuamente haciendo), el conjunto de las relaciones del ámbito, la calidad ambiental, así como la historia y la inercia de las relaciones. De los tres ámbitos o elementos del enfoque de justicia relacional, la socialidad -en la medida en que representa más cercana y concretamente a la relación en sí misma - es el que resulta más humanamente perceptible, pudiendo presentar una mejor o peor calidad ambiental (más en detalle, Márquez Prieto, 2010). Es decir, una percepción en positivo de la relación que, en la medida en que ayude y beneficie a las personas, podremos considerarla más humana; o al contrario, si la relación produce un ambiente negativo.Así pues, si identificamos la calidad ambiental con una situación relacional de justicia (justi- 
cia en la relación), o un clima de confianza y cooperación, como ilustrativamente se ha descrito en el juego de la caza del ciervo, surge la cuestión sobre lo que contribuye a que el juego relacional sea uno y no otro. La calidad ambiental relacional depende, en primer lugar, de la orientación hacia la justicia de los sujetos de la relación - en lo que puede denominarse el nivel intencional de la justicia relacional-, y, en segundo lugar, de forma determinante, de la respuesta que la propia relación ofrezca; situándonos ya así en el nivel o plano emergente de la justicia relacional, caracterizado por un cierto margen de contingencia. En ese margen de contingencia adquiere relevancia - entre otros elementos- el hecho de que suele ser un gran número de relaciones - formales o no, jurídicas o no- el que contribuye a generar un determinado grado de calidad ambiental relacional. Una institucionalidad que responda al paradigma de red facilita a su vez una buena socialidad de red, por el efecto de reciprocidad global que puede verse propiciado por determinadas condiciones. En concreto, el efecto que, sobre la calidad ambiental relacional produce una red de relaciones -como hemos expuesto en Repensar la justicia social (Márquez-Prieto, 2008: 124-125), y con apoyo en un estudio previo de Luigino Bruni (2006: 113-190)-, depende directamente de la densidad y diversidad de las estrategias y actitudes presentes en la misma. En este sentido, Teubner afirma que

[...] las redes expresan ciertamente reciprocidad, pero de un tipo diferente. La diferencia fundamental entre una red y un contrato multilateral -en el sentido técnico restringido de do ut des - viene constituida por una reciprocidad generalizada y omnicomprensiva que espera un retorno, no de la transacción inmediata, sino de relaciones sociales, sustancialmente indeterminadas, de largo plazo y socialmente difusas (Teubner, 2005: 87-88).

\section{LA BUENA FE Y EL CÍRCULO VIRTUOSO DE LA JUSTICIA RELACIONAL}

Luigino Bruni (2012: 29) propone una visión amplia y actual de la idea de virtud, en base a las siguientes notas características:

1) la virtud es una disposición (o un rasgo) del carácter de un individuo, relativa a un específico dominio;

2) es algo tendencialmente estable en el tiempo [...];

3) es susceptible de ser cultivada por el individuo y por los otros con los que interacciona, y por tanto el individuo es responsable de la mis$\mathrm{ma}$; 
4) una vez cultivada, la virtud ayuda a la persona a alcanzar la excelencia en un determinado dominio [...];

5) lo que se obtiene por medio de la virtud (carácter, comportamiento, hábito...) debe obtener la aprobación y la simpatía de los otros, en el interior de ese determinado dominio;

6) en fin, es la disposición el objeto de la aprobación y de la simpatía de los otros, y no primariamente los resultados de las acciones, si bien entre los dos niveles existe una relación circular.

Es a esa relación circular (entre la disposición, intención, comportamiento... y el resultado de las acciones) a la que en este trabajo se alude con la expresión "círculo virtuoso». En la medida en que Bruni se refiere a la virtud como «característica de las relaciones, y no sólo de los individuos» (2012: 32-33), se pretende aquí presentar con claridad la justicia relacional como una categoría relacional de justicia que, en caso de estar presente en la relación, explica el círculo virtuoso de la justicia. Es decir - de forma paralela a las notas propuestas como caracterizadoras de la virtud, entendida ésta como dinámica circular-, si las partes orientan su comportamiento recíproco hacia la justicia, si la institucionalidad (normativa, contractual...) se orienta igualmente a la justicia, puede emerger continuamente una relación o socialidad cuyo fruto sea una calidad ambiental de justicia - en dicha relación-, que, a su vez, servirá de criterio e inspiración para que, de nuevo, tanto la reciprocidad como la institucionalidad, se orienten hacia la justicia. En otro caso, por la falta de orientación a la justicia, por la falta de adaptación entre los tres elementos de la justicia relacional, encontraremos un círculo vicioso, caracterizado por vacíos de justicia (injusticia relacional). En otros trabajos hemos presentado el enfoque de justicia relacional como técnica capaz de identificar y explicar los distintos vacíos de justicia en la relación (Márquez-Prieto, 2010). En este lugar, debido al intento de precisar algo más la categoría de justicia relacional, como categoría capaz de explicar la existencia o ausencia de justicia en la relación y el cierre del círculo virtuoso en los términos expuestos por Luigino Bruni, sólo se aludirá a un concreto vacío de justicia: la ausencia o ignorancia del valor intrínseco, como característica humana (precisamente porque Bruni conecta su idea de virtud con la motivación intrínseca).

El profesor Bruni (2012: 179 y ss.) recurre al juego de la confianza (como muestran las siguientes figuras), para explicar el valor de la motivación intrínseca $(\varepsilon)$, entendida como sinónimo de comportamiento no obligatorio o excedencia, don, que va más allá de lo obligatoriamente exigido, de acuerdo con una práctica que, en un determinado ámbito, se acepta como idónea 
para alcanzar la excelencia, es decir, una motivación intrínseca virtuosa (independientemente de las razones de dicha motivación).

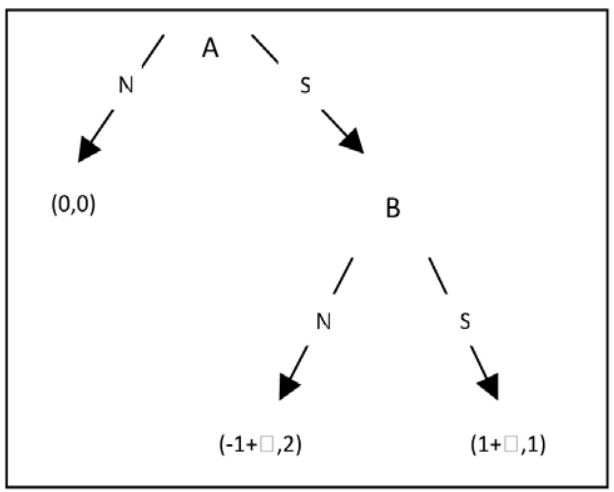

Se trata de un juego secuencial, en el que aparecen, mediante un diagrama en árbol, los pagos atribuidos a ambos jugadores (A y B) dependiendo de si cada uno de ellos decide confiar en el otro (s) o no confiar (n). Sencillamente la relación no llegará a establecerse, porque A, que es quien juega primero, valorará que su confianza en B dará a éste a su vez la opción de una respuesta de no colaborar (no confianza) o colaborar (en caso de que confíe). Y la razón que lo disuadirá de confiar en B será que para éste, el pago de la no confianza será mayor (es decir, 2) que el de la respuesta de confiar a su vez en A (1). La situación cambia, y pasa a establecerse realmente la relación, si al jugador $\mathrm{A}$ se le atribuye un pago adicional $(\varepsilon)$ en caso de que $\mathrm{A}$, por su motivación intrínseca, esté dispuesto a cooperar con $\mathrm{B}$, a pesar de que $\mathrm{B}$ no responda afirmativamente. En ese caso $\varepsilon$ ha de ser superior a 1 .

Como se deduce de la figura, sea cual sea la respuesta de B que A pueda prever, la relación se establecerá porque (como $\varepsilon>1$ ) A siempre obtendrá un pago superior a 0 (que es su pago correspondiente para cuando no coopera).Ahora bien, como también Bruni pone de manifiesto, a pesar de todo A se hace dependiente de $B$, porque sigue quedando en disposición de B la posibilidad de responder o no positivamente. Es decir, «la we-rationality [racionalidad del nosotros] se convierte en este caso en importante porque añade a la gratuidad una versión más fuerte de socialidad» (Bruni, 2012: 188). Lo que implica que la cooperación social, el consenso, el mantenimiento de la relación, de acuerdo con un clima de confianza (o buena fe), exige que cada uno de los jugadores tenga en cuenta, no su recompensa individual, sino la recompensa del conjunto (la suma de recompensas). 
La racionalidad del nosotros - aun considerando siempre las enormes limitaciones y dificultades de la convivencia humana- es precisamente la mentalidad de partida del Derecho. El consenso constituye, en efecto, la opción tradicional del Derecho, que parte de la necesidad y de la posibilidad de actuar de buena fe. Es decir, el Derecho no sólo se ha considerado tradicionalmente a sí mismo como un método de solucionar conflictos, sino también como un instrumento de regulación de la conducta y de construcción de expectativas (Teubner, 2005: 133), lo que permite atender, procurar y sostener el consenso.

Resulta crucial, en este sentido, la «confianza en la palabra», como ha propuesto Alain Supiot (Supiot, 2005: 174-175), quien reivindica el valor incalculable de la confianza entre los sujetos en su trato jurídico (ámbito de reciprocidad), así como el valor de la palabra. La atención a estos dos elementos se traduce así, para dicho autor, en la confianza en la palabra; es decir, en el trato humano, en la interacción personal y directa, que genera una dinámica que no puede ser sustituida por el contrato ni por la ley. En similares términos podríamos decir que nada puede sustituir el valor incalculable de una relación jurídica viva. Es ésa la realidad relacional a la que atiende la categoría de justicia relacional, considerando conjuntamente - en su dinámica interactiva - sus tres elementos (institucionalidad, reciprocidad y socialidad).

Interesa aclarar que el enfoque de la justicia relacional alude a una realidad que no puede considerarse en absoluto novedosa (aunque sí su denominación o categorización como tal), teniendo en cuenta que ya en el Derecho romano podía percibirse con claridad la atención por parte de los juristas al elemento de socialidad, en un contexto en el que quedaban evidenciados también los otros dos aspectos. En este sentido, la diferencia entre reciprocidad e institucionalidad remite en Roma al dualismo entre el ius y la lex, que no sólo se corresponden con dos fuentes jurídicas, sino igualmente con dos paradigmas de soberanía. ${ }^{11}$ El binomio entre la jurisprudencia (de los juristas) y la legislación (del comicio, del pretor, del senado) tuvo una cierta continuación a través del Digesto, haciendo renacer en la Edad Media la complementariedad entre «norma y vida, entre reglas y comportamientos» (Schiavone, 2005: 14, 29). El ius representaba propiamente la reciprocidad (aunque, en cuanto fuente de producción de reglas obligatorias, no era ajeno a la institucionalidad). Se trataba de una reciprocidad horizontal, paritaria - entre los grupos familiares, re-

11 «[... la ciudad se encontró de hecho ante sí, igualmente definidas, dos hipótesis diversas de organización normativa y de regulación social: dos modelos alternativos de soberanía, podríamos decir. Uno fundado sobre el paradigma, específicamente romano, del ius; el otro sobre aquél, griego y mediterráneo, de la lex. No es arriesgado sostener que la confrontación habría tenido consecuencias incalculables: de ella habría dependido la invención de la «forma derecho» en el transcurso de Occidente» (Schiavone, 2005: 76). 
presentados por los paterfamilias-, expresada, como convicción colectiva, a través de un saber sapiencial y aristocrático -que respondió a una labor, primero de los sacerdotes, y después de los juristas-, lo que había ido dando lugar a un rito capaz de regular las relaciones sociales a nivel privado (Schiavone, 2005: 50, 86, 115). Fue la presión plebeya la que logró introducir en Roma el paradigma de soberanía democrática a través de la lex, que representaba una relacionalidad fuerte, pública y escrita (contrariamente al ius, transmitido como un saber secreto, casuístico y oral). Pero la misma lex preveía el recurso al ámbito de socialidad (populum). Así, la Ley de las xII Tablas contemplaba el necesario pronunciamiento de las asambleas populares (como criterio de legitimación, de justicia soberana, o de conciencia ciudadana) para algunos supuestos más delicados producidos por la aplicación de la lex. Así, se requería un proceso ante el comitium centuriatum para una condena a muerte, o se preveía un recurso ante la asamblea popular (provocatio ad populum, a través de la Lex Valeria de provocationis) para cuando se consideraba que el magistrado había ejercido de forma arbitraria su imperium (Santalucía, 1998: 45 y ss.). E igualmente el elemento de socialidad era lo que podía justificar un atemperamiento de la aplicación rígida del propio ius. La máxima summum ius, summa iniuria expresaba, en base al principio de equidad (aequum) la necesidad de adoptar una medida más flexible si la aplicación rígida del ius civile pudiese producir un resultado injusto (ius civile et iniquum), atendida la socialidad concreta del caso (los valores o intereses en juego). Era precisamente la «sustancia de las relaciones», como ha advertido Schiavone (2005: 126 ss., especialmente 129), el criterio para considerar si la aplicación del ius podía resultar inicua desde el ámbito de la socialidad.Así puede incluso afirmarse que la socialidad poseía para los juristas romanos un sentido de soberanía jurídica. Más allá de la soberanía patriarcal que para el ius representaban los paterfamilias, los juristas «lograron evitar caer prisioneros de un vínculo demasiado estrecho entre conocimiento jurídico y actuación política» (Schiavone, 2005: 37), aferrándose a la idea de ciudadanía civil (o privada), acudiendo continuamente a la indagación de esas «relaciones y proporciones invisibles, entre las cosas y entre las personas», de esa «sustancia de relaciones» $(35,129)$, como criterio último (o soberano) de la juridicidad en cada caso. ${ }^{12}$

12 «[... el terreno propio del ius: un campo que la mentalidad romana no habría nunca identificado con el poder del rey, para reservarlo a la acción de cualquier modo soberana de los patres, directamente dependiente sólo de vínculos religiosos no coincidentes con la esfera de la realeza: el germen originario - el núcleo generativo- de la regulación autónoma de una trama "privada» de la vida comunitaria (también privus es una palabra arcaica), donde eran elaborados los presupuestos familiares y patrimoniales de la ciudadanía, irreductiblemente distinta del orden político; un aspecto que no encontramos en estos términos en otra sociedad mediterránea, destinado a formar un rasgo que habría tenido una continuidad extraordinaria» (Schiavone, 2005: 50). 


\section{PRECISIONES FINALES SOBRE LA JUSTICIA RELACIONAL}

Llegado es, pues, el momento de precisar algo más el sentido de la justicia relacional como categoría concreta de justicia, de forma coherente con lo hasta ahora expuesto, en base a las siguientes anotaciones:

- la justicia relacional no se refiere a ninguna de las categorías concretas de justicia individualizadas ya de forma clásica desde Aristóteles (como la justicia distributiva o conmutativa, aunque las incluye y presupone), sino a lo que éste consideraba justicia en sentido universal, o virtud perfecta, llamada así por dos razones: por englobar todas las virtudes y porque no se usa solamente para consigo mismo, sino para con los demás; en síntesis, la obligación de actuar conforme a la virtud para con el otro (2001: 1130 b);

- tradicionalmente existe en el Derecho una denominación que expresa de forma específica esa rectitud, virtud genérica, o motivación intrínseca en la actuación respecto a los demás: la buena fe. Podría decirse que la buena fe traduce el contenido positivo de la justicia relacional, en términos principalmente de confianza.Así, la relación, las relaciones (la socialidad), ofrecen confianza en un funcionamiento virtuoso de la dinámica relacional; la norma, lo institucional, apunta al reforzamiento de la confianza, protegiendo e incentivando al que actúa de buena fe; y la reciprocidad, la actuación ex bona fides, no sólo requiere dar continuamente el paso (a veces el primer paso), sino que requiere también fiarse, asumiendo ese riesgo, como acto de voluntad. Se corresponde con la motivación intrínseca $(\varepsilon)$ en el juego de la confianza; ${ }^{13}$

- puede decirse también que la buena fe se corresponde con el estándar de excelencia, según esa nueva forma de entender la virtud en la era de los bienes comunes (a la que alude Bruni), que puede permitir percibir la justicia en la relación -cuando existe- como un verdadero bien común;

- la justicia relacional, como característica o cualidad de la relación, se conecta, pues, no sólo con la motivación intrínseca -o virtud-, sino que constituye un verdadero círculo virtuoso (en la medida en que se mantiene presente y no se desvirtúa).Así, volviendo al juego de la confianza, no sólo es preciso que, en base a la motivación intrínseca, se dé el paso de confiar y establecer la relación, sino que tiene también que existir una respuesta, lo cual, a su vez, incluye no sólo la perfección de

13 Un tratamiento sobre la buena fe desde el Derecho romano, respecto al enfoque de justicia relacional, es abordado por Caro-Gándara (2013: 11-67). 
contratos, la consolidación individual de la virtud, la aprobación social o estabilización de comportamientos (es decir, la dinámica entre reciprocidad e institucionalidad); la respuesta implica también el empuje de la socialidad, de la cultura, en la medida en que, compartiendo el valor de la motivación intrínseca, constituya inspiración e incentivo para una reciprocidad y una institucionalidad virtuosas.

$\mathrm{Si}$, por el contrario, no existe una relación de verdadera justicia, sino un vacío de ella, entramos en un círculo vicioso, que puede ser explicado por la falta de orientación a la justicia de alguno de los tres elementos, o por la falta de adaptación entre sí. En este sentido, interesa aludir, ya en fase final -y como se había anunciado - a un concreto vacío de justicia, al que también alude Luigino Bruni: la paradoja del trabajo por cuenta ajena en la empresa. Dicha paradoja consiste en que, si bien los dirigentes empresariales y los trabajadores saben que un trabajo verdaderamente fructuoso, eficiente y eficaz, es el que, más allá de lo estipulado como obligatorio, incorpora una implicación realmente personal del trabajador, la organización económica y jurídica en el ámbito empresarial deja fuera de la valoración, la contabilización y la contratación la parte más importante de la prestación laboral: el cómo, la calidad personal y motivacional del trabajo. Esa parte, en la medida en que no se puede comprar, porque pertenece al ámbito de la gratuidad, permanece conscientemente ignorada. En palabras de Bruni, «las cláusulas y las características de los contratos de trabajo se paran exactamente antes de entrar en las cosas que verdaderamente cuentan en una relación humana de trabajo, que dura ya años y que se nutre de todas aquellas dimensiones que ningún contrato puede ni prever ni especificar» (Bruni, 2012: 193).

La interpretación de esta paradoja quizá contribuya a precisar un poco más cómo la justicia relacional, como categoría que caracteriza o cualifica a la relación, constituye, cuando está presente, un círculo virtuoso, en el que los distintos elementos de la relación, en la medida en que se orientan hacia la justicia, se retroalimentan entre sí. La paradoja del trabajo por cuenta ajena muestra un círculo vicioso en el que se pretende jugar al juego de la confianza sin tener en cuenta la motivación intrínseca. Como se ha visto, en dicho juego no se establece la relación si no existe una verdadera motivación intrínseca. Esto significa que la relación establecida, como relación laboral, no puede constituir una relación genuina. Tanto si la falta de la motivación intrínseca es achacable al jugador $\mathrm{A}$, como si quien la ignora es el jugador $\mathrm{B}-\mathrm{O}$ ambos-, la relación transcurre impulsada únicamente por la institucionalidad, de forma automatizada, pero no de forma humana (no hay reciprocidad ni socialidad verdaderamente personales), porque el vacío de justica consis- 
te en la ignorancia del elemento intrínseco de la motivación humana (algo parecido al trato con una máquina de café con instrucciones de voz grabadas). La única forma de salir de ese círculo vicioso, de esa relación desvirtuada, es iniciar una dinámica de reciprocidad fáctica, aun no contratada, que, yendo más allá de lo obligatorio, recupere, de forma interactiva -aunque sea implícitamente-, un trato humano recíproco verdaderamente satisfactorio desde el punto de vista humano.

\section{BIBLIOGRAFÍA}

ARISTÓTeles (2001): Ética a Nicómaco [Introducción, traducción y notas de José Luis Calvo Martínez], Madrid, Alianza Editorial.

BAIRD, D. G. y otros (1994): Game Theory and the Law, Cambridge-usa y Londres, Harvard University Press.

BRUNI, L. (2012): Le nuove virtù del mercato nell'era dei beni comuni, Roma, Città Nuova.

- (2006): Reciprocità. Dinamiche di cooperazione, economia e società civile, Milán, Bruno Mondadori.

CARO-GÁndARA, R. (2013): «En la secular búsqueda europea de un paradigma de justicia contractual: el enfoque de justicia relacional» en SÁnCHÉz-Lorenzo, S. (ed.) (2013): Derecho contractual comparado. Una perspectiva europea y transnacional. Estudios, Madrid, 2. ${ }^{a}$ ed., Civitas.

CORTÁZAR, R. (1997): «Non-Redundant Groups, the Assurance Game and the Origins of Collective Action», Public Choice, vol. 92, pp. 41-53.

Donati, P. (2006): Repensar la sociedad, Madrid, Ediciones Internacionales Universitarias SA.

García-Ruiz, P. (2000): «Antecedentes de la Socioeconomía: individuo y comunidad en el proyecto sociológico de Parsons», Anthropos (monográfico sobre Socioeconomía), 188, pp. 111-123.

- (2006): «Presentación» en Donati, P. (2006): Repensar la sociedad, Madrid, Ediciones Internacionales Universitarias.

Gunvitch, G. (1932): L'idée du droit social, París, Sirey (reimpresión 1972, París, Scientia Verlag Aalen).

Hardin, G. (1968): «The Tragedy of the Commons», Science, 162, pp. 12431248.

Jassen, M. (2001): «On the principle of coordination», Economics and Philosophy, vol. 17, 2, pp. 221-234. 
Márquez-Prieto, A. (2008): Repensar la justicia social. Enfoque relacional, teoría de juegos y relaciones laborales en la empresa, Pamplona,Thomson-Aranzadi.

- (2010): Calidad ambiental de las relaciones laborales, Granada, Comares.

Martínez-Coll, J. C. (1986): Bioeconomía, Málaga, Servicio de Publicaciones de la Universidad de Málaga.

Parsons, T. (1937): The Structure of social action, Nueva York, McGraw Hill.

Rousseau, J. J. (1754, 1985): Discours sur l'origine et les fondements de l'inégalité parmi les hommes, París, Bordas.

Santalucia, B. (1998): Diritto e proceso penale nella antica Roma, Milán, Giuffrè.

Schiavone, A. (2005): Ius. L'invenzione del diritto in Occidente, Turín, Einaudi.

SKYRMs, B. (2007): La caza del ciervo y la evolución de la estructura social, Barcelona, Melusina.

Stanford Encyclopedia of Philosofy (2004): «Games Theory and Ethics» [http:// plato.stanford.edu/entries/game-ethics/].

Supiot, A. (2005): Homo juridicus. Essai sur la fonction antropologique du Droit, París, Seuil.

Teubner, G. (2005): La cultura del Dirittonell'epoca della globalizzazione. L'emergere delle costituzioni civili, Roma,Armando Editore. 